\title{
ON DISEASE OF THE MASTOID BONE.
}

BY

\author{
W. B. DaLBY, M.B. Cantab., F.R.C.S., \\ AURAL SURGEON TO ST. GEORGE'S HOSPITAL.
}

(Received November 26th, 1878-Read January 14th, 1879.)

THE objects of the following communication are, to add another case to those already recorded, in which a purely local irritation excited malignant disease in a patient with no discoverable predisposition towards it, and in which epithelioma in its progress eroded and destroyed large portions of bone; to call attention to the instances in which the probable fatal effects of inflammation may be averted by perforating the mastoid cells; and to illustrate how the whole of the temporal bone may, in a child, be destroyed by ulceration and the patient survive.

In March of this year, Agnes $\mathbf{S}$-, a married woman, æt. 32, whilst picking her left ear with a hair-pin, ruptured the tympanic membrane, and soon after the accident came under my notice as an out-patient of St. George's Hospital. With the exception of this lesion she was, in all respects, in good health. The rupture did not heal, and in a few days, from the fistulous opening thus established, there was a purulent discharge. A month later, after an attack of pain in the ear vol. LXII. 
which was followed by facial paralysis of that side, I again examined the ear and found a polypoid mass filling up the cavity of the tympanum, the membrane having by this time quite gone. She now came into the hospital; I removed the polypoid growth, and the pains in the ear, which had previously been considerable, passed off. Her stay in hospital, on this occasion, was three weeks.

On July 31st, when she again applied for relief and was admitted, she stated that she had suffered from no further pain until within five weeks, when acute pain in the ear came on, and soon afterwards the parts over the mastoid process became swollen and tender. Two weeks ago, she said, the skin over the swelling broke down and a little bloody matter was discharged. The ragged wound at that time observable was the result, and from this wound had been coming ever since a quantity of watery, very foulsmelling discharge. The skin over the mass was bluish, the tissues were infiltrated, and the edges of the wound were everted and hard. In short, the disease was to all appearance malignant. There were no enlarged glands, neither was there any history, to be obtained, of cancer in her family. No loose bone could be detected, although a large surface of bone was exposed. From this time she rapidly wasted; the wound increased in size until it formed a large cavity discharging most offensive matter, and she died on November 12th, from exhaustion without any head symptoms or hæmorrhage. The following is the description of the post-mortem appearance given by Mr. Ewart, the curator of the museum. Beyond what is mentioned there was no disease throughout the body.

"The ulcer, which was nearly circular, had a diameter of three inches. It was deeply excavated in the centre in the shape of a funnel; the diameter of this central hole was two inches; it extended forward and inwards to a depth of two inches in the direction of the long axis of the petrous bone, and terminated close to the lining membrane of the mouth at a point just internal to the inferior maxillary articulation. The surface of the cavity was foul, dirty green in colour, it was 
partly formed by the white necrosed remains of the petrous portion and the sloughing cartilage of the jaw. At the periphery of the ulcer the colour was that of proud flesh. The surface was everywhere irregular and knobby ; this tuberculation was especially marked at the lower part where there was a considerable amount of fibrous and œdematous thickening. No distinct tumour could be made out. The bone was extensively destroyed and absorbed. The mastoid process, the external meatus, and tympanum, as also parts of the petrous portions of the temporal bone had entirely disappeared. Viewed from the intracranial aspect the petrous surfaces were normal, but the dura mater having been stripped, it was found that the jugular foramen had become enlarged by ulceration. The inferior maxillary articulation was disorganised, and the articular surfaces exposed. The bone surrounding the gap showed no new growth infiltration, but the granulations, whilst spreading as a thin layer over the squamous portion of the temporal bone had noticeably eroded the subjacent bone.

Under the microscope, the diseased tissue consisted mainly of vascular meshes containing numerous small cellular islands, the smallest of these groups consisting of from four to six cells, presented ill-defined characters and intermediate forms between epithelial cell and fibre. The larger groups were plainly epithelial ; no intercellular substance intervened between the cells; the cells had a large oval nucleus containing nucleoli or granules, and otherwise resembled epithelial cells. A few birds' nests were found.

The case is worth attention, as forming one of a series in which the mastoid bone has become the seat of malignant disease, and in which in every instance there has been a local irritation preceding the malignant growth. Thus, with one exception in all the cases I find recorded (there are six in all) the tympanic cavity had been in a state of suppuration for a long or short period before there was any evidence of malignant growth. In two of the cases reported by Sir William Wilde, the one a woman aged 50, and the other a man aged 55, there had been a discharge from the ear 
for many years, and for several years in the case of a boy aged 7. The same condition was present in the examples related by $\mathrm{Mr}$. Toynbee. In the exception referred to, although the local irritation did not take the form of a discharge from the ear, there was a distinct local cause assignable, as the subject, a boy, in being knocked down by a cab, received a violent blow on the side of the head. As he is said to have suffered from great pain in the ear, followed by facial palsy, it seems probable that even in this case the tympanum was the seat of inflammation for some time before the appearance of malignant disease. The case was reported by Mr. Cooper Forster, in the 'Pathological Transactions' for 1850. Like other morbid growths of the middle ear, malignant tumours appear to arise from the mucous membrane which lines the tympanum, and the rule seems to be that this mucous membrane is the seat of suppuration for some time before malignant disease begins. Now this rule also applies to cases of polypus, and did apply in the case of a round-celled sarcoma, which is reported in my lectures on diseases of the ear. In regard to the origin of malignant disease without any apparent predisposition, six out of the seven cases form a small collection for consideration. Let them be placed with some thousands of cases in which from similar causes the tympanic cavity becomes inflamed, and the membrane is ruptured. In some the perforation will heal; in others the opening will remain through life, the tympanum discharging purulent matter more or less, but no growth arising; in others the local irritation of the discharging surface will induce polypus from the tympanum; in a few others will induce by discharge passing through the external canal, bony enlargements, the so-called exostoses. In the early days of the new growths how little (within our knowledge) divides the malignant from the simpler form. In the very case under notice, there was a period when from the cavity of the tympanum there was seen to be what to all appearance was a simple polypoid growth. After this was removed there was another period which may be described as one of quiet, 
lasting for over six weeks, and during which no active disease seemed to be going on. Indeed, the malignant aspect was not shown until after an attack of inflammation of the mastoid cells, which before this attack had remained healthy. The unknown force which determines the character of the disease remains unknown, and it is but a poor explanation to say that the subject must have had a predisposition to cancer, seeing how easily cases of malignant disease might be brought forward in abundance, in which equally, as with this woman, no such predisposition is to be found. To show that without doubt cancer is frequently inherited, of course in no way disposes of the question.

The resemblance of this case to one where an ordinary ulcer of the leg takes on (to use a common expression) malignant growth in the form of epithelioma, which finally erodes and destroys portions of the tibia is very striking. In both a suppurating surface exists for some time before the simple granulations are replaced by epithelioma.

To pass on to the inflammatory disease of the mastoid bone-although the course of inflammation in general proceeds from the pharynx up the Eustachian tube into the tympanum, and so on to the mastoid cells, these cavities within the mastoid process occasionally become primarily inflamed, whilst throughout the whole course of the inflammation the tympanic cavity remains healthy. In each and every case, however, the principal points of importance to decide are, when and in what manner should an escape be artificially (i.e. surgically) provided for pus within the mastoid cells. These two questions are full of interest, and upon correct answers given to them the life of the patient often depends. I suppose it will be admitted that, if in all cases where there is pas within the mastoid cells no attempt were made to provide an egress for it, a certain number of patients would die from further complications before the process of ulceration had effected the desirable opening. Under what circumstances then should the mastoid cells be perforated? When should the case be allowed to proceed 
till the bone has so far become softened as to permit of the opening of the so-called abscess by the knife? An answer to the first inquiry may $I$ think be found from considering the following case, which came under my notice in the summer of the present year :

On the 10th June a young man in vigorous health was, whilst on board ship, without any apparent cause, attacked with acute inflammation in the right tympanum. The pain was so intense, that he did not sleep (he said, in narrating his case) for five days; at the end of which period, the tympanic membrane gave way and allowed the escape of a purulent discharge from the external ear. Although this was followed by great immediate relief, at intervals he continued to suffer from pain in the head, till the 25th, on which day I saw him for the first time; on the previous evening he had a slight shivering fit. The discharge was then profuse, the constitutional disturbance was great, and he had lost over a stone in weight since the beginning of his illness. There was very slight tenderness on deep pressure over the mastoid process, but no redness or œdema. The treatment pursued consisted simply of leeches over the mastoid process, hot fomentations, and the ear was syringed frequently with warm water.

Matters continued much the same until the evening of the 9 th of July, when he had a severe rigor. On the next day prolonged and deep pressure over the mastoid process caused considerable pain, and, for the first time, very slight œedema was noticeable. On this day (under ether) I made an incision over the mastoid bone through the periosteum (the bone was healthy), and bored through the bone into the cells. This took some time to accomplish as the bone was very thick. When the opening into the mastoid cells was completed, about half a drachm of thick pus escaped through the wound. For the first time since the 10th of June he slept well through the night. On the following day the complete communication between the tympanic cavity and the mastoid 
cells, was shown by the facility with which he could (the mouth, nostrils, and external ear being firmly closed) blow through the external wound. In doing so a quantity of pus was expelled. Twice during each day he cleared the tympanum and mastoid cells in this way, and the discharge, now escaping through the wound, ceased to come from the ear. Suffice it to say that he recovered without a bad symptom.

No surgeon who saw this case would, I believe, doubt the propriety of the proceeding which was adopted. Mr. Warrington Haward, who was good enough to help me at the operation, was completely in agreement upon this point. It was happily not done too late, but should it not have been done before? I cannot but reflect that I have never, either in my own practice or in that of others, known the mastoid cells to be opened before it was needed; but that I have seen, and I suspect that many others have done so, cases in which this operation might have been performed with advantage. I believe that when the bone is unusually thick, we are apt to be deceived by the absence of superficial tenderness and early œdema. In children, of course, the case is widely different ; superficial tenderness, redness, and odema, are early symptoms, and often are present before there is any pus in the mastoid cells. In such instances $I$ have sometimes cut down to the bone, broken into the cells (this with children is, of course, readily done), and been disappointed at not finding an immediate flow of pus. Disappointed without reason, inasmuch as on the next day pus has escaped in plenty through the wound, the tympanum has been relieved, the acute symptoms have subsided, and the death of the bone has been avoided.

The point, however, which I would strongly urge is that, when in all these cases an incision has been made on to the mastoid process and the bone is found to be healthy, the opening into the mastoid cells should be completed; and that when, in the case of adults, a distinct rigor has taken place, this proceeding should not be delayed; because if the symptoms of what is termed mastoid abscess are waited for, the 
patient will at times die from cerebral abscess, meningitis, or pyæmia.

When, in the above case, the discharge secreted by the lining membrane of the tympanic cavity had an escape provided for it through the external wound, and so ceased to be poured out through the perforation of the tympanic membrane, the hearing power at once increased, showing that the tympanum shared the improved condition of the other parts. The chance of inflammation proceeding upwards to the cranial cavity was thus at once lessened. Briefly, then, in similar circumstance there are few reasons which will warrant delay, and many which should urge decision.

These remarks will of course equally apply, whether the process of inflammation owes its origin to any of the exanthemata, or to so-called catarrhal inflammation of the middle ear.

After all that has been said of the necessity for prompt measures in these cases, it seems out of place to contemplate even the possibility of what in actual practice is common enough, viz. the death of the whole or portion of the mastoid process, especially as these dead parts remove themselves by the almost unaided efforts of ulceration. The disasters to the auditory apparatus, to the portio dura, and to life, which occasionally form rude interruptions to this process, also occur frequently enough to require no comment except to say that, it is with a view of occasionally avoiding such interruptions, I venture to advocate surgical aid.

The subject of the third case, L. E. B-, was a child, æt. 1 year and 8 months, who had a discharge from the ear with perforation of the nuembrane since the age of 6 months, and pieces of bone had from time to time come from the external auditory canal. It is only necessary to say that, at the time she came under my observation, on 30th March, 1875, there was a profuse purulent discharge from the right ear, facial palsy of that side, total deafness, and a large fluctuating swelling over the mastoid process; that after the swelling had been freely laid open the dead bone under. 
neath was not found to be loose, but that a month later, I removed with my finger and thumb a large piece of dead bone which included in its mass, what to all appearance, was all that remained of the temporal bone. After this the wound healed; the external ear sank deeply into the pit which was left; the child recovered its health, and is now well with the exception of the loss of nervous power which has been referred to. 